\title{
9. \\ Gli Excerpta Planudea, Pietro Patrizio e la tradizione storiografica occidentale
}

\author{
Laura Mecella
}

DOI: http://dx.doi.org/10.7359/820-2017-mace

ABSTRACT: The paper focuses on the so-called Excerpta Planudea, a collection of excerpts on Roman history (from the Republic to the reign of Gratianus), written in Constantinople by the monk Maximus Planudes (about 1265-1305). According to an old conjecture, its last excerpt derives from the historia of Peter the Patrician / Anonymus post Dionem, and it would be an evidence for the now lost Annales of Virius Nicomachus Flavianus senior. A thorough analysis of the fragment and of the tradition flowed into the Excerpta Planudea rules out this hypothesis and confirms that the most probable ending of Peter the Patrician's bistoria were the years of Constantius II.

KEYWORDS: Anonymus post Dionem, Excerpta Planudea, Gratianus, Peter the Patrician, Virius Nicomachus Flavianus senior - Anonymus post Dionem, Excerpta Planudea, Graziano, Pietro Patrizio, Virio Nicomaco Flaviano senior.

Pietro Patrizio non fu, a rigore, autore cui Antonio Baldini dedicò ricerche specifiche; e tuttavia il suo ruolo appare centrale per affrontare un tema che allo studioso fu invece particolarmente caro, ovvero l'esistenza e la natura degli Annales di Virio Nicomaco Flaviano senior - vexata quaestio ancor oggi al centro del dibattito scientifico. Non intendo, in questa sede, ripercorrere analiticamente i termini della questione ${ }^{1}$; basti dire che mi trova sostan-

1 Recente mise au point sugli Annales flavianei in Vitiello 2014, che pur respingendo l'ipotesi di una loro identificazione con la Historia Augusta (tenacemente sostenuta da Ratti 2010, 217-223, 239-248, 252-276; Ratti 2012, 139-148 e 154-164; Ratti 2014, 425 428), ritiene che la raccolta latina di biografie - a suo dire ascrivibile, secondo quanto già proposto da Festy 2007, alla mano di Virio Nicomaco Flaviano iunior -, ne abbia in gran parte raccolto l'eredità; per un'equilibrata valutazione della documentazione epigrafica relativa ai due Nicomachi cf. inoltre Girotti 2015. Completamente diversa la ricostruzione di Cameron 2011, capp. 17-18 e 20, che data la composizione della Historia Augusta tra il 375 e il 380 e ritiene gli Annales nulla più di una «trivial epitome», con scarsa o nulla 
zialmente concorde la ricostruzione che, sulla scorta degli studi avviati da Bleckmann e Paschoud, Baldini propose dei rapporti tra l'opera del magister officiorum Pietro e la tradizione latina identificata negli Annales flavianei, ricostruzione in estrema sintesi riassumibile in due punti: identità di Pietro Patrizio e del cd. Anonymus post Dionem, e suo utilizzo degli Annales (direttamente o tramite una traduzione greca) per tutto il segmento di storia post-erodianeo (per la sezione precedente, infatti, Pietro dimostra di attingere quasi esclusivamente a Cassio Dione ed Erodiano) ${ }^{2}$. In tale prospettiva, il corpus di 215 frammenti (dal 40 a.C. al 358 d.C.) che si ottiene sommando la tradizione adespota degli Excerpta historica Constantiniana con i frammen-

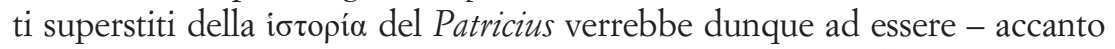
all'Epitome de Caesaribus, ai resti della seconda edizione della storia di Eunapio, e forse alle Res Gestae di Ammiano Marcellino ed alla Historia Augusta - un importante vettore di trasmissione dei perduti Annales flavianei ${ }^{3}$.

Se infatti non vi sono ragioni per dubitare di una consultazione diretta da parte di Pietro della produzione greca diffusa nell'Oriente tardoanti$\mathrm{CO}^{4}$, è pur vero che alcuni frammenti della sua storia presentano evidenti tracce di una matrice occidentale: nella Costantinopoli di età giustinianea si riverbera un ricco patrimonio culturale di origine latina, che sulle vicende di III-IV secolo consegna alla memoria storica orientale tradizioni di chiara ascendenza senatoria ${ }^{5}$. In questo quadro, e nell'incertezza che ancora regna sulla natura degli Annales, al nome di Nicomaco Flaviano senior quale possibile fonte di Pietro deve piuttosto essere attribuito un valore

eco presso la posteriore storiografia. Per l'arco cronologico presumibilmente abbracciato dall'opera cf. infra, n. 24.

2 Tra i suoi tanti lavori dedicati al tema, mi limito a segnalare la densa trattazione in Baldini 2000, 97-177, e Baldini 2005; cf. inoltre Bleckmann 1992 e più recentemente, con ampia discussione delle tesi di Cameron, Bleckmann 2015 e Paschoud 2012, 359-388, in partic. 369-380 e 385-386 (cui si rinvia per la menzione dei suoi precedenti lavori). Sulla dipendenza di Pietro da Cassio Dione vd. ora Roberto 2016.

3 Gli Excerpta de legationibus ascrivono a Pietro Patrizio 19 frammenti dal principato di Tiberio al Cesarato di Giuliano in Gallia (358 d.C.), mentre il lessico segueriano Пврі

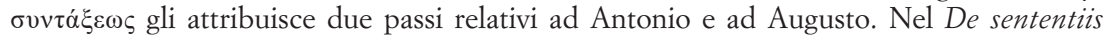
troviamo poi, da un lato, un gruppo di 158 escerti adespoti, di ispirazione dionea ma non riconducibili direttamente allo storico bitinico, dall'altro 36 frammenti, anch'essi anonimi, dal regno di Massimino il Trace all'età di Costantino (gli Excerpta post Dionem di Angelo Mai, appunto): secondo la convincente ricostruzione proposta a partire da Niebuhr 1829, XXIV, e successivamente ripresa, per non citare che i principali contributi degli ultimi decenni, da Bleckmann 1992, Martolini 2010 e Banchich 2015, questi brani sono tutti riconducibili alla mano di Pietro.

4 Sul punto vd. ora Zecchini c.d.s.

5 Oltre alla bibliografia cit. supra, n. 2, cf. Bleckmann 2010 (per gli echi ammianei nel cd. Giovanni Salmasiano) e, per un approfondimento di queste tematiche, Mecella c.d.s. 
antonomastico, atto a definire l'inconfutabile continuità di tradizioni fra la storiografia occidentale di spiriti filosenatori di III-IV secolo e la prima letteratura bizantina ${ }^{6}$. Continuità che, peraltro, si riscontra anche per il periodo successivo, quando la «fronda storiografica» (come la definì Baldini) alla cristianizzazione voluta da Teodosio si riflette, da un lato, in Olimpiodoro e Zosimo, dall'altro, nella Historia Romana di Memmio Simmaco, da qui passando poi a Marcellino comes, Cassiodoro, e Giordane ${ }^{7}$.

Di questa catena di trasmissione tra l'Occidente latino e l'Oriente greco l'opera di Pietro è dunque anello fondamentale, poiché raccogliendo parte della riflessione storica elaborata a Roma nella cerchia dei Simmachi-Nicomachi ne ha garantito una pur pallida sopravvivenza presso la successiva cronografia bizantina, come hanno dimostrato le diverse analisi condotte sugli scritti di Simeone Logoteta, Giovanni Zonara, Giorgio Cedreno e Teodoro Scutariote ${ }^{8}$. I debiti di questi autori nei confronti della produzione storiografica di età giustinianea sono stati infatti indagati almeno parzialmente, raggiungendo talora risultati importanti ${ }^{9}$; in questo quadro minore attenzione, invece, è stata rivolta ad un altro importante testimone della scrittura storica di età imperiale e tardoantica, ovvero i cd. Excerpta Planudea.

Si tratta di un gruppo di più di trecento escerti di storia romana, da Romolo all'età di Graziano, conservato principalmente in cinque manoscritti miscellanei, di cui il più autorevole, nonostante la ricostruzione stemmatica sia ancora dibattuta, sembra il Laurentianus gr. pl. 59.30 (ff. 30r-47v), al-

6 Sui pericoli insiti in un'ossessiva «caccia al nome» quando si parla di tradizioni storiografiche piuttosto che di opere dalla fisionomia ben definita metteva già in guardia Baldini 2000, 66-68, in riferimento però all'attribuzione della Enmannsche Kaisergeschichte.

7 Cf. Baldini 1999; Baldini 2004, 241-258, 267-272; Baldini 2007; Baldini 2010. Nel presente volume vd. poi il contributo di T. Stickler.

8 A Teodoro Scutariote va ascritta la cronaca convenzionalmente nota come Synopsis Sathas dal nome del suo primo editore, che la pubblicò dal Marcianus gr. 407, ff. $8^{\mathrm{r}}-138^{\mathrm{v}}$ (Sathas 1894). È merito di Tocci 2015 aver fornito un'aggiornata edizione critica della prima fase compositiva dell'opera, i cd. Chronica, estesi da Adamo fino alla morte di Alessio I Comneno (e per noi documentati dall'autografo Vaticanus gr. 1889, ff. $17^{\mathrm{v}}-32^{\mathrm{v}}$ ); a questa prima stesura Teodoro avrebbe poi apportato diverse modifiche, dando così vita alla Synopsis. Di quest'ultima è ancora in preparazione da parte di Tocci una nuova edizione; nell'attesa, essa continuerà dunque ad essere citata secondo il testo di Sathas 1894. Sullo statuto del genere cronografico a Bisanzio vd. ora in generale Mariev 2015.

9 Per non menzionare che i principali contributi dell'ultimo trentennio (cui si rimanda per un approfondimento sulla storia degli studi), va ricordato, in primis, il già citato Bleckmann 1992, soprattutto per Simeone Logoteta (una delle cui redazioni dell'opera nella più antica letteratura è attribuita al cd. Leone Grammatico) e Zonara; per Cedreno, si dispone ora di una nuova edizione critica preceduta da una sintetica ma efficace introduzione (Tartaglia 2016, I, 13-25); per Teodoro Scutariote vd. Tocci 2015, 8*-115*, in partic. $83 *-95 *$ sulle sue fonti. Per una discussione sull'eredità di Pietro nella posteriore cronachistica cf. anche Mecella c.d.s. 
lestito nello scriptorium del monaco Massimo Planude. Tale raccolta non conosce una tradizione autonoma, ma si configura come parte di una più

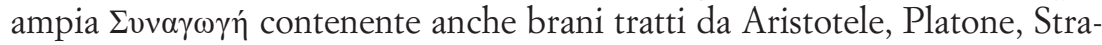
bone, Pausania, Eliano, Ateneo, Sinesio e Giovanni Lido, oltre che da varie opere di contenuto cristiano ${ }^{10}$. Eccellente esempio di quella «cultura della

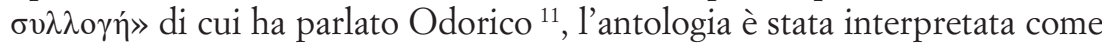
un manuale preparatorio alle lezioni che si tenevano nella scuola di Planude: sul finire del XIII secolo, Massimo istituì a Costantinopoli un importante centro di studi, e principalmente all'attività didattica sembra dedicata la silloge nella quale i frammenti di storia romana sono conservati. Sarebbe impossibile, in questa sede, tracciare anche un rapido schizzo della gigantesca personalità di Planude, intellettuale dagli interessi disparati e non avulso dalla politica; basti dire che la configurazione della raccolta riflette due delle principali caratteristiche del suo magistero: l'estrema cura per la conservazione del testo (l'attività filologica del monaco e della sua scuola è ben nota) e, rara avis nel panorama bizantino dell'epoca, l'amore per il mondo latino (di cui Planude conosceva molto bene anche la lingua) ${ }^{12}$.

Nonostante la raccolta planudea si riveli dunque di grande interesse non soltanto come testimone di opere più antiche, ma anche per una migliore comprensione della temperie culturale costantinopolitana nella prima età paleologa, essa è stata finora indebitamente trascurata. Solo nel 2015 è uscita la prima edizione completa degli excerpta platonici a cura di Lorenzo Ferroni, ed un'operazione analoga sarebbe ora auspicabile anche per la sezione di storia romana. Essa rimane infatti in gran parte inedita: l'editio princeps del cardinale Angelo Mai interessa parzialmente solo 90 frammenti su ca. 335, e i successivi studi si sono perlopiù concentrati sui primi 44 brani dell'antologia ${ }^{13}$. Un'edizione dell'intero corpus di storia

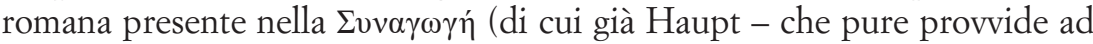
aumentare la selezione dei brani pubblicati - lamentava l'assenza) rappresenta dunque un vivo desideratum della ricerca ${ }^{14}$; e nell'attesa di adeguati

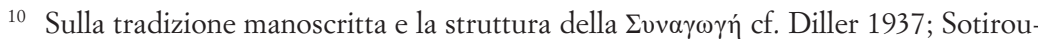
dis 1989, 202-213; Roberto 2005, CI-CX; Mariev 2008, 21*-24*, e soprattutto Ferroni 2011 e Ferroni 2015, 22-43.

11 Odorico 1990.

12 Non potendo riportare un'esaustiva bibliografia sull'argomento, mi limito a rimandare alla recente sintesi in Mecella - Roberto 2017, 138-148 (con precedente letteratura).

13 Cf. Mai 1827, 527-555; Mommsen 1872; Piccolomini 1874, 105-116; Haupt 1879a, 36-57; Haupt 1879b, 443-446; Boissevain 1895, CXI-CXXIII; Kugéas 1909, 126 146; Roberto 2005, CX-CXVII.

14 Haupt 1979a, 54 e 47-50, per il gruppo di frammenti inediti riportati dal Parisinus gr. 1409 (sec. XIV-XV) e relativi alla sezione «dionea» via Xifilino. L'auspicio di «una

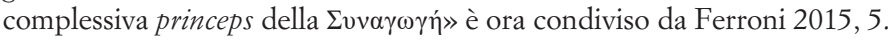


strumenti filologici e di un'indagine sistematica di tutti i frammenti della raccolta, anche le osservazioni che seguono mantengono inevitabilmente un carattere di provvisorietà.

Principalmente sulla base dei pionieristici contributi della filologia tedesca di fine Ottocento - inizio Novecento, è possibile ricostruire così l'articolazione del florilegio di argomento storico: se il primo brano rimanda all'opera di Costantino Manasse (di cui torneremo a parlare tra poco) e il secondo a quella di Giovanni d'Antiochia, i frammenti 3-4 derivano dalla tradizione di Peanio (che, com'è noto, alla fine del IV secolo tradusse in greco il Breviarium di Eutropio), mentre il frammento 5 si mostra come una contaminazione di informazioni tratte dal De magistratibus di Giovan-

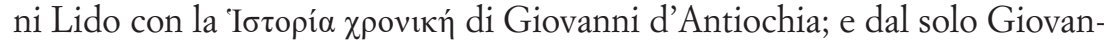
ni d'Antiochia derivano gli escerti 6-44 (sul periodo che da Romolo arriva alla III guerra mitridatica). Per i frammenti dal nr. 45 in poi, fino all'anno 229 d.C. si individuano principalmente due tradizioni: quella di Cassio Dione filtrata dall'epitome di Xiphilino, e ancora reminiscenze eutropiane mediate da Peanio; Inmaculada Pérez Martín ha recentemente ipotizzato che tali materiali siano in parte derivati a Planude dalla lettura del codice dell'Athos Iviron 812, di cui la studiosa anticipa la datazione al XII secolo e dove sono appunto conservati passaggi da Eutropio-Peanio, Giovanni d'Antiochia e Xifilino ${ }^{15}$. Questi due filoni non sembrano tuttavia esaurire la ricchezza delle tradizioni confluite in Planude: corrispondenze con la cronaca di Manasse si riscontrano anche in questa parte (in brani relativi al filosofo Atenodoro e all'imperatore Tiberio) ${ }^{16}$ e significativo, in proposito, appare anche l'escerto relativo alla condotta di Caligola, che come ha ben mostrato Kugéas si rivela inequivocabilmente tratto dalle Antiquitates Iudaicae di Giuseppe Flavio (XIX 204-208), non sappiamo tuttavia attraverso quale ramo di trasmissione ${ }^{17}$. È evidente, dunque, che solo un'indagine sistematica dell'intero corpus planudeo, e non soltanto della porzione sinora nota, potrà aiutare a chiarire la complessa genesi della raccolta.

15 Pérez Martín 2015, 179-189: la studiosa riconosce la mano di Planude in alcune delle glosse presenti nel codice, dimostrando così da parte dello stesso Planude la conoscenza diretta di almeno una porzione del testo di Xifilino, precedentemente messa in dubbio, ad esempio, da Haupt 1879a, 46, 53-54 e 57, che pensava piuttosto all'esistenza di una fonte comune tra Xifilino e Planude (posizione poi però da lui stesso ritrattata in Haupt 1879a, 295).

16 Cf. Piccolomini 1874, 111 (senza tuttavia indicazione della fonte); Haupt 1879a, 52-54, 293-294, e Haupt 1879b, 444; Kugéas 1909, 146.

17 Kugéas 1909, 137 e 146 (laddove Haupt 1879a, 55-56, si era invece limitato ad affermarne l'estraneità alla tradizione dionea). 
In ogni caso, Peanio viene seguito anche per il periodo post-dioneo fino all'età di Costanzo Cloro, mentre incerta è l'origine del brano conclusivo della silloge planudea (relativo a Graziano), in cui alcuni hanno voluto individuare proprio la mano dell'Anonymus post Dionem. Nel pubblicare per la prima volta alcuni passi nel secondo volume della Scriptorum veterum nova collectio, Angelo Mai parlava genericamente di post Dionem excerpta ex Anonymo bistorico, senza identificarlo con quel Continuator Dionis del De sententiis che noi riteniamo essere Pietro Patrizio ${ }^{18}$; è stato soltanto Ludwig Dindorf, nella sua edizione dionea, seguito qualche anno dopo da Theodor Mommsen, a lasciar trapelare l'idea che gli escerti di Planude po-

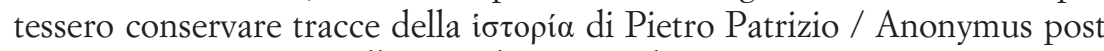
Dionem, senza tuttavia illustrare le ragioni di questa convinzione:

Illud mibi superest dicendum, quod nempe Planudes continuationem quoque Dionis ab anonymo auctore curatam, aeque ac Constantinianus eclogarius legebat. (Dindorf 1865, 233)

Dass die die Kaisergeschichte betreffenden Excerpte des Titels de sententiis aus derselben Quelle geflossen sind wie die planudischen, ist evident; wie denn auch Dindorf, ohne sonst den Sachverhalt zu erkennen, wenigstens die drei nachdionischen den Maximianus, Constantin [ ic $^{19}$ ] und Gratian betreffenden Bruchstücke bei Planudes richtig mit den entsprechenden Fragmenten des constantinischen Titels combinirt hat. (Mommsen 1872, 90)

L'autorevolezza dei proponenti ha fatto sì che l'ipotesi si riverberasse sulla letteratura scientifica quasi con grado di certezza, determinando una communis opinio tuttora attestata.

Indubbiamente, singolari appaiono le scelte di Planude nel confezionare i suoi appunti per le lezioni e la ratio che ne è alla base spesso rimane oscura. Da rilevare, innanzitutto, l'assenza di Zosimo almeno per il periodo post-dioneo, dal momento che il Vaticanus gr. 156, codex unicus della

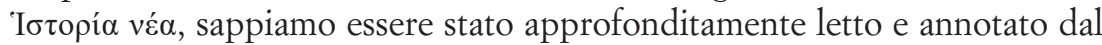
monaco bizantino: gli studi di Forcina prima, e di Bianconi poi, hanno dimostrato come alla sua mano vadano ricondotti alcuni marginalia del manoscritto, rilevando un grande interesse nei confronti del testo zosimiano ${ }^{20}$. Planude polemizza con Zosimo sottolineando la magnanimità di Costantino e rifiutando l'idea che l'imperatore abbia fondato Costantinopoli per

18 Mai 1827, 567-568. Cloro.

19 In realtà gli escerti (cit. infra, n. 25) sono relativi a Massimino il Trace e Costanzo

20 Forcina 1987, 35-36, 42-43, 83-93 (che identifica Planude solo con la cd. «mano D»); Bianconi 2005, 434-436, che invece attribuisce a Planude anche le annotazioni della cd. «mano E» ai ff. $38^{\mathrm{v}}, 48^{\mathrm{r}}, 74^{\mathrm{v}}$. 
ostilità nei confronti dei Romani; mentre in relazione a Zos. I 29.3, dove si ricorda la ricostruzione delle mura da parte degli Ateniesi sotto Valeriano per difendersi dalle invasioni, il monaco ricorda come a nulla sarebbe potuta valere la protezione di Atena: è evidente che al momento di redigere la glossa egli aveva già letto l'ultimo libro dell'opera, dove Zosimo attribuisce proprio alla dea la salvezza della città dall'assedio di Alarico (Zos. V 6.1-2). Come ha scritto Forcina «l'interesse, anche se polemico, nei confronti dello storico pagano è tale che vale la pena di rileggerlo e riprenderlo in mano più volte»; e proprio nel tono anticristiano dell'opera la studiosa individua uno dei possibili motivi per l'esclusione dalla silloge planudea ${ }^{21}$. Si potreb-

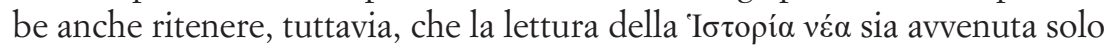
dopo la composizione della $\Sigma v v \alpha \gamma \omega \gamma \eta$, impedendone dunque l'utilizzo in quella sede.

Inoltre, se già la volontà di dedicare ampio spazio alla storia della repubblica romana (generalmente trascurata nelle sintesi bizantine) appare eccentrica, ancora più arduo è cogliere le motivazioni per un arresto con l'età di Graziano; vi si potrebbe scorgere, forse, l'intento di valorizzare la storia dell'imperium Romanum prima della partitio d'età teodosiana. Nel B $\alpha \sigma \iota \lambda \iota \kappa o ̀ \varsigma ~ \lambda o ́ \gamma o \varsigma$, un panegirico composto nel 1295 in onore di Andronico II e del figlio e coreggente Michele IX, Planude celebra la Roma sul Tevere come Urheimat dei due imperatori, presentando il capostipite della dinastia, Michele VIII, come un novello Costantino ${ }^{22}$ : emerge chiaramente, da questo pezzo d'occasione, la volontà di esaltare l'impero romanobizantino come unico vero impero universale, ancora a capo dell'intera ecumene. Non si può pertanto escludere che questa idea sia stata anche alla base della sua epitome sulla storia di Roma; ma l'ipotesi rimane, allo stato attuale della documentazione, purtroppo indimostrabile.

Poiché comunque, in questo tipo di raccolte, le grandi cesure corrispondono generalmente anche ad un cambiamento di fonte, alcuni studiosi hanno ritenuto che la stessa Vorlage seguita da Planude si arrestasse con Graziano; in questo quadro il nome dell'Anonymus post Dionem, sulla scorta delle intuizioni di Dindorf e Mommsen sopra citate, è apparso un buon candidato, nella convinzione che anche la sua opera terminasse, nel solco del modello flavianeo, con il $383^{23}$.

21 Forcina 1987, risp. 83 e 90.

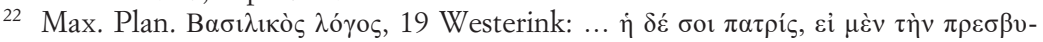

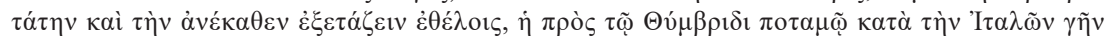

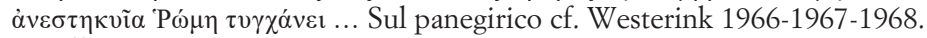

${ }_{23}$ Cf. e.g. Haupt 1879a, 48 e 56; Baldini 2000, 148 e 151. 
Tale ricostruzione poggia tuttavia su una serie di presupposti aprioristici: stabilita la conclusione degli Annales flavianei con l'epoca di Graziano

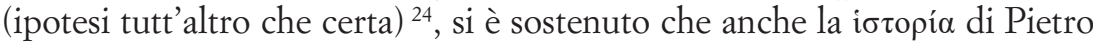
finisse con la stessa data; su questa base si è dunque identificata in Pietro Patrizio / Anonymus post Dionem una delle fonti di Planude, considerando che anche la selezione di storia romana operata dal monaco bizantino si arrestava appunto con Graziano.

$\mathrm{Al}$ centro della discussione è l'ultimo frammento degli Excerpta Planudea. Degli unici tre brani relativi al periodo post-severiano che sono stati assegnati all'Anonymus post Dionem, i primi due, relativi a Massimino il Trace e Costanzo Cloro, si mostrano in realtà derivati dalla tradizione di Eutropio-Peanio già significativamente attestata nella raccolta, lasciando dunque escludere ulteriori intermediazioni ${ }^{25}$.

Di più difficile contestualizzazione è invece il brano conclusivo dell'antologia:

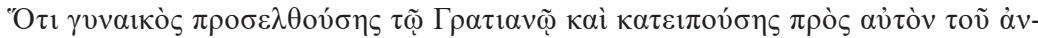

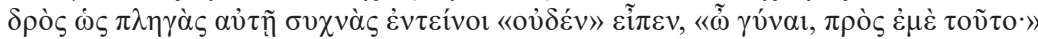

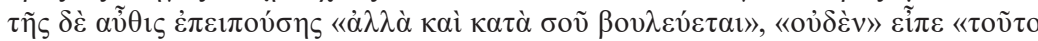
$\pi \rho \grave{\varsigma} \sigma \delta \dot{\sharp}$.

Poiché una donna si avvicinò a Graziano accusando il marito di picchiarla ripetutamente, quello rispose: «Donna, questo non mi riguarda». E poiché quella incalzava: «Ma sta meditando qualcosa anche contro di te», l'imperatore rispose «Questo non ti riguarda».

Si tratta effettivamente di un aneddoto paragonabile ai tanti motti di spirito presenti nel De sententiis, ma nessuna positiva evidenza permette di ricondurlo al nome di Pietro Patrizio / Anonymus post Dionem: non solo esso non compare tra i frammenti trasmessi dall'enciclopedia costantiniana, ma nemmeno la tradizione del Logoteta (pur nelle sue versioni interpolate), di Cedreno e di Zonara, che molto risente dell'eredità di Pietro, ne conserva memoria. La stessa ricostruzione del contesto evenemenziale appare chimerica: anche un pur vago collegamento con l'agguato di Andragazio che con-

24 Così come sostenuto per esempio da Festy 1997, 472-475, e Paschoud 2000², LXILXII. Di diverso avviso Zecchini 1993, che propende per un arresto al 388/389.

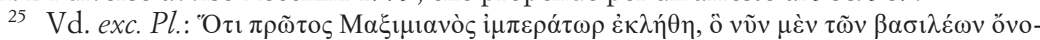

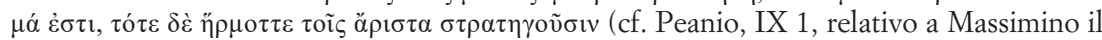

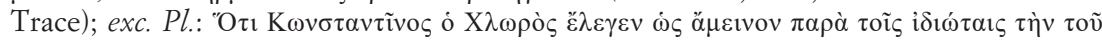

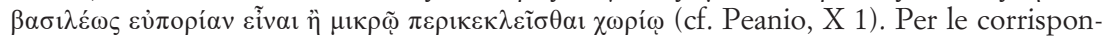
denze tra questi frammenti e il testo di Peanio cf. già Pirogoff 1873, 90-91; Piccolomini 1874, 109 e 116; Haupt 1879a, 54-55; Boissevain 1901, 749; Kugéas 1909, 146. 
dusse alla morte dell'imperatore si rivela del tutto arbitrario ${ }^{26}$. L'Epitome de Caesaribus, Ammiano Marcellino ed Eunapio - al pari di Pietro presumibili latori della tradizione flavianea che sarebbe alla base del racconto - tacciono dell'episodio e non riportano altre insidie minacciate contro l'imperatore.

Il passo si ritrova soltanto, peraltro in forma pressoché identica, in Costantino Manasse, autore di una cronaca universale in versi (decapentasillabi) dalla creazione del mondo al regno di Niceforo III Botaniate (10781081) ${ }^{27}$. Ai vv. 2407-2413 Lampsides leggiamo:

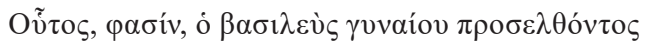

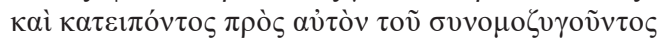

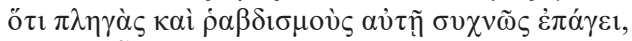

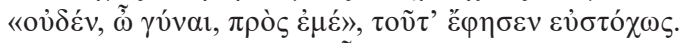

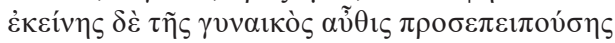

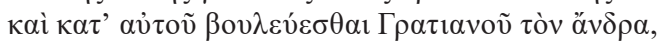

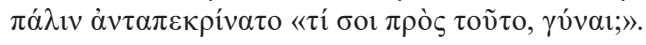

L'imperatore, dicono, poiché gli si era avvicinata una donna accusando presso di lui il marito che le infliggeva ripetutamente percosse e bastonate, disse sagacemente: «Donna, questo non mi riguarda». E poiché quella disse ancora che il marito stava meditando qualcosa contro Graziano stesso, quello replicò: «Donna, forse che questo ti riguarda?».

La strettissima correlazione tra i due passaggi era già stata notata da Boissevain ${ }^{28}$. Anche sulla base degli altri frammenti assimilabili alla tradizione di Manasse di cui si è parlato in precedenza, se ne ricava, dunque, o che Massimo Planude abbia direttamente utilizzato la cronaca di Costantino (che l'autore fosse certamente noto a Planude lo dimostra, nella stessa $\Sigma v v \alpha \gamma \omega \gamma \eta$, la ripresa del romanzo di Manasse intitolato Aristandro e Callitea), o che abbia attinto alla sua stessa fonte, come sostenuto da Kugéas ${ }^{29}$. In ogni caso,

26 Andragazio, magister equitum sotto l'usurpatore Magno Massimo, uccise Graziano a tradimento facendogli credere che la moglie fosse giunta nell'accampamento e lo stesse aspettando: l'imperatore, precipitatosi da lei, trovò invece il suo assassino pronto a colpirlo. L'episodio è riportato da Soz. h.e. VII 13.8-9; Socr. h.e. V 11.7-8; Sym. Log. 94.2 Wahlgren; Cedr. 334.2 Tartaglia. Menzionano l'esecuzione senza descrivere lo stratagemma Zos. IV 35.6; Ruf. h.e. II 14; Io. Ant. fr. 279 Roberto; Zon. XIII 17 (III 226, 1l. 14-16 Dindorf); Theod. Scut. syn. 661-662 Sathas (cf. chron. II 105 Tocci). Sul personaggio cf. PLRE I, Andragatbius, 3, 62-63.

27 Sintesi sull'autore e le principali caratteristiche dell'opera in Lampsides 1988 e Lampsides 1996, I, XI-CLIX; Nilsson 2006; Nilsson - Nyström 2009; Kiapidou 2009 (principalmente per la sezione tra Costantino ed Eraclio); Rhoby 2014.

28 Boissevain 1901, 750. Da Manasse desume l'aneddoto anche Michele Glica (474, 11. 12-17 Bekker).

${ }^{29}$ Kugéas 1909, 135-136, 138, 145-146; cf. anche de Boor 1893, 202-203, secondo cui Planude avrebbe piuttosto attinto ad un rifacimento in prosa della cronaca di Manasse. 
indipendentemente dalla relazione tra i due, la chiave di volta del problema appare costituita da questa Manassesquelle in grado di riportare informazioni non altrimenti attestate. Manasse condivide con Simeone Logoteta e Cedreno, entrambi latamente debitori della tradizione di Pietro Patrizio (forse attraverso la cd. Epitome ${ }^{30}$ ), soltanto la lode per le straordinarie capacità di Graziano nel tiro con l'arco (tanto da far sembrare che le sue frecce avessero una mente) ${ }^{31}$ : la sua fonte deve quindi aver lavorato su un materiale comune a questo filone, ma attingendo ad un ulteriore ramo di trasmissione ${ }^{32}$.

La natura ibrida della cronaca di Manasse, a metà tra il racconto storico e la narrazione novellistica (la forte patina letteraria dell'opera è già evidente nella scelta della forma poetica) rende ancor più scivoloso il terreno d'indagine; e forse la pista più interessante resta finora quella aperta da Mai nell'editio princeps del frammento planudeo. Pur ammettendo l'impossibilità di rintracciarne l'origine, il cardinale ne rilevava una vaga attinenza con quanto riportato da Eunapio in Excerpta de sententiis 55 ${ }^{33}$ : dopo aver lamentato l'assenza di informazioni per il regno di Graziano, e la difficoltà di delineare un profilo dell'imperatore, lo storico di Sardi ricordava comunque come fosse possibile imparare dagli esempi che seguivano, su cui tutti concordavano e che nessuno, tra coloro che avevano l'abitudine di diffondere pettegolezzi, negava ${ }^{34}$. Non si potrebbe dunque escludere, secondo

Di derivazione diretta aveva invece parlato Haupt 1879a, 291-295, e Haupt 1880. Per i

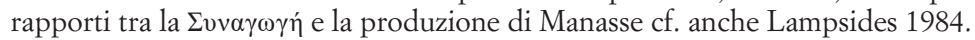

30 Si tratta di una fonte perduta ipotizzata da Edwin Patzig per giustificare alcune corrispondenze tra le diverse cronache dell'età mediobizantina. Di questa Epitome nel $\mathrm{X}$ secolo sarebbero esistite due redazioni: una Redazione $A$, confluita nelle varie versioni in cui circolò il testo del Logoteta, ed una (più ampia) Redazione $B$, cui invece avrebbe attinto Cedreno: cf. Patzig 1894; Patzig 1896; Patzig 1897; Praechter 1897; Serruys 1907; Bleckmann 1992 (che ha persuasivamente dimostrato come alla base dell'Epitome, per la storia profana del III - inizio IV secolo, non vi sarebbe stata la tradizione salmasiana di Giovanni d'Antiochia, secondo quanto ipotizzato da Patzig, ma, appunto, Pietro Patrizio); Brecht 1999, 52 ss. Dal canto suo, il nuovo editore di Cedreno, pur ricordando che le corrispondenze tra quest'ultimo e il Logoteta potrebbero anche essere dovute o ad una derivazione diretta dal Logoteta, oppure alla mediazione del cd. Pseudo-Simeone (l'anonima cronaca tramandata dal Parisinus gr. 1712 che costituisce l'intelaiatura di base del racconto di Cedreno), non nega l'influenza dell'Epitome su queste Hauptquellen di Cedreno, e non modifica, pertanto, nella sostanza, il quadro di riferimento: Tartaglia 2016, I, 19-23.

31 Cf. Man. 2414-2415 Lampsides; Sym. Log. 94.3 Wahlgren; Cedr. 334.3 Tartaglia. Da Manasse o dalla sua fonte desumono l'informazione anche Michele Glica (474, 11. 10 12 Bekker) e Theod. Scut. 62, 11. 23-24 Sathas (cf. chron. II 97 Tocci).

32 Per i rapporti tra Manasse e il cd. Leone Grammatico, ovvero Simeone Logoteta, oltre alla bibliografia cit. supra, n. 27, cf. anche Rhoby 2014, 394 e 399.

33 Mai 1827, 279-280 e 567-568.

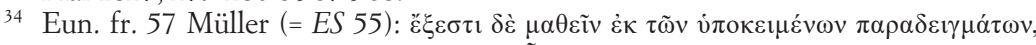

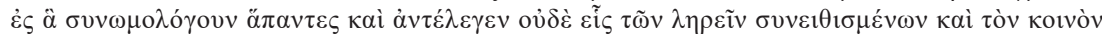

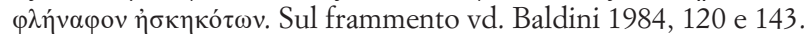


Mai, che il passo riportato da Manasse e Planude fosse inserito proprio in questa selezione di exempla contenuti nella seconda edizione della storia eunapiana e che erano mirati ad illustrare il carattere dell'imperatore.

E tuttavia, sebbene a priori simile matrice appaia verosimile, in verità ci troviamo costretti ad ammettere la nostra totale ignoranza sull'origine del brano e sulle fonti di informazione della Manassesquelle per questa sezione $^{35}$; quel che pare di potersi affermare con un buon grado di verosimiglianza è soltanto la sua estraneità alla tradizione di Pietro Patrizio. Poiché

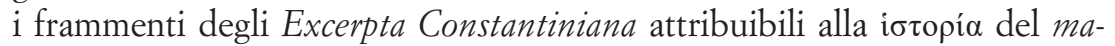
gister si arrestano con il 358, non abbiamo alcuna ragione di ritenere che l'opera proseguisse oltre l'età di Costanzo $\mathrm{II}^{36}$. Come già sottolineato in apertura, con questo non si vuole affatto negare l'influenza della tradizione occidentale, diciamo pure «flavianea», sulla sua storia; ma nell'incertezza che ancora circonda l'arco cronologico abbracciato dagli stessi Annales e nell'assenza, come si è tentato di dimostrare, di ulteriori testimonianze, ritenere che essa si fosse spinta fino agli anni '80 del IV secolo appare quantomeno arbitrario.

In conclusione: l'analisi dell'opera di Pietro Patrizio / Anonymus post Dionem costituisce un passaggio non soltanto legittimo, ma necessario ai fini di una migliore intelligenza dell'eredità della produzione storica (non soltanto latina) di età tardoantica nella letteratura della media e tarda età bizantina. E se i risultati dell'esame appaiono perlopiù modesti e incerti, è bene sempre tenere a mente il motto con cui lo stesso Baldini chiudeva la magistrale ricerca confluita nel volume Storie perdute: molto spesso, in questo tipo di studi, «val meglio la caccia che la preda» ${ }^{37}$.

35 Legittima, ma allo stato attuale della documentazione puramente ipotetica, è la proposta di Haupt 1879a, 295-296, di identificare l'Urquelle del passo con Giovanni d'Antiochia. Certamente i rapporti tra quest'ultimo e la cronaca di Manasse rappresentano un campo di studi fecondo e meritevole di ulteriori approfondimenti.

36 Ho affrontato in altra sede il problema del perché di questa scelta, apparentemente anomala, legando la questione alla genesi dell'altro scritto di Pietro Patrizio, il Пврì

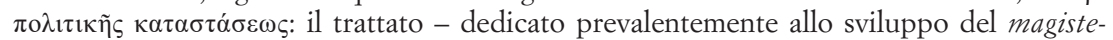
rium officiorum - potrebbe essere stato originariamente concepito come costola della più ampia opera storica, per assumere solo in seguito la forma di un componimento auto-

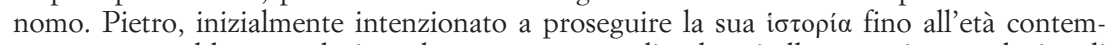
poranea, potrebbe aver deciso ad un certo punto di volgersi alla trattazione esclusiva di quella magistratura di cui egli stesso fu, per ben 26 anni, illustre rappresentante; in questo quadro, una conclusione del proprio racconto storico con il regno di Costanzo II appare decisamente funzionale, poiché proprio a questo imperatore si dovette un'energica azione di sistematizzazione del programma di riforme paterno: cf. in dettaglio Mecella c.d.s.

37 Baldini 2000, 243. 


\section{BIBLIOGRAFIA}

Baldini 1984

Baldini 1999

Baldini 2000

Baldini 2004

Baldini 2005

Baldini 2007

Baldini 2010

Banchich 2015

Bianconi 2005

Bleckmann 1992

Bleckmann 2010

Bleckmann 2015

Boissevain 1895

Boissevain 1901
A. Baldini, Ricerche sulla storia di Eunapio di Sardi. Problemi di storiografia tardopagana, Bologna 1984.

A. Baldini, Un'ipotesi su una tradizione occidentale post-flavianea, in F. Paschoud (ed.), Historiae Augustae Colloquium Genevense, Bari 1999, 13-31.

A. Baldini, Storie perdute (III secolo d.C.), Bologna 2000 .

A. Baldini, Ricerche di tarda storiografia (da Olimpiodoro di Tebe), Bologna 2004.

A. Baldini, Considerazioni in tema di «Annales»e di «Historia Augusta», in G. Bonamente - M. Mayer (edd.), Historiae Augustae Colloquium Barcinonense, Bari 2005, 15-46.

A. Baldini, Tra «Historia Augusta» e «Storia Romana» di Q. Aurelio Memmio Simmaco, in G. Bonamente - H. Brandt (edd.), Historiae Augustae Colloquium Bambergense, Bari 2007, 9-34.

A. Baldini, Un nucleo di fronda storiografica nel senato di Roma tra IV e VI secolo, in G. Bonamente R. Lizzi Testa (a cura di), Istituzioni, carismi ed esercizio del potere (IV-VI secolo d.C.), Bari 2010, 31-49.

Th.M. Banchich, The Lost History of Peter the Patrician: An Account of Rome's Imperial Past from the Age of Justinian, London 2015.

D. Bianconi, La biblioteca di Cora tra Massimo Planude e Niceforo Gregora. Una questione di mani, Segno e testo 3 (2005), 391-438.

B. Bleckmann, Die Reichskrise des III. Jabrbunderts in der spätantiken und byzantinischen Geschichtsschreibung. Untersuchungen zu den nachdionischen Quellen der Chronik des Johannes Zonaras, München 1992.

B. Bleckmann, Der salmasische Johannes Antiochenus. Ein Versuch zur Bestimmung seines Profils für die Geschichte der Spätantike, in L. Galli Milić N. Hecquet-Noti (edd.), Historiae Augustae Colloquium Genevense, Bari 2010, 51-61.

B. Bleckmann, «Last pagans», Source Criticism and Historiography of the Late Antiquity, Millennium 12 (2015), 103-116.

U.Ph. Boissevain (ed.), Cassii Dionis Cocceiani Historiarum Romanarum quae supersunt, I, Berolini 1895.

U.Ph. Boissevain (ed.), Cassii Dionis Cocceiani Historiarum Romanarum quae supersunt, III, Berolini 1901. 
Brecht 1999

Cameron 2011

de Boor 1893

Diller 1937

Dindorf 1865

Ferroni 2011

Ferroni 2015

Festy 1997

Festy 2007

Forcina 1987

Girotti 2015

Haupt 1879a

Haupt 1879b

Haupt 1880

Kiapidou 2009

Kugéas 1909

Lampsides 1984

Lampsides 1988

Lampsides 1996
St. Brecht, Die römische Reichskrise von ibrem Ausbruch bis zu ibrem Höhepunkt in der Darstellung byzantinischer Autoren, Rahden 1999.

Al. Cameron, The Last Pagans of Rome, Oxford 2011.

C. de Boor, Römische Kaisergeschichte in byzantinischer Fassung. III. Die Salmasischen und Treuschen Exzerpte. Manasses, ByzZ 2 (1893), 195-211.

A. Diller, Codices Planudei, ByzZ 37 (1937), 295-301.

L. Dindorf, Dionis Cassii Cocceiani Historia Romana, V, Lipsiae 1865.

L. Ferroni, I manoscritti della $\Sigma v v \alpha \gamma \omega \gamma \eta ́$ planudea, SCO 57 (2011), 327-353.

L. Ferroni, Maximi Planudis e Platonis dialogis compendia, Bologna 2015.

M. Festy, Le début et la fin des «Annales» de Nicomaque Flavien, Historia 46 (1997), 465-478.

M. Festy, L'«Histoire Auguste» et les Nicomaques, in G. Bonamente - H. Brandt (edd.), Historiae Augustae Colloquium Bambergense, Bari 2007, 183-195.

A.M. Forcina, Lettori bizantini di Zosimo. Le note marginali del cod. Vat. gr. 156, Milano 1987.

B. Girotti, Nicomaco Flaviano, «historicus disertissimus»?, Hermes 143 (2015), 124-128.

H. Haupt, Über die Herkunft der dem Dio Cassius beigelegten planudischen Excerpte, Hermes 14 (1879), 36-64; 291-297.

H. Haupt, Neue Beiträge zu den Fragmenten des Dio Cassius, Hermes 14 (1879), 431-446.

H. Haupt, Zum planudischen «Continuator Dionis», Hermes 15 (1880), 160.

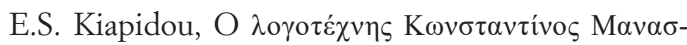

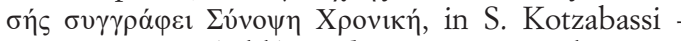
G. Mavromatis (edd.) Realia Byzantina, Berlin 2009, 57-66.

S. Kugéas, Analekta Planudea, ByzZ 18 (1909), 106146.

O. Lampsides, «Collectanea Planudea» und die «Chronike Synopsis» von Konstantinos Manasses, ByzZ 77 (1984), 1-2.

O. Lampsides, Zur Biographie von K. Manasses und zu seiner Chronike Synopsis (CS), Byzantion 58 (1988), 97-111.

O. Lampsides (ed.), Constantini Manassis Breviarium chronicum, I-II, Athenai 1996. 
Mai 1827

Mariev 2008

Mariev 2015

Martolini 2010

Mecella c.d.s.

Mecella - Roberto 2017

Mommsen 1872

Niebuhr 1829

Nilsson 2006

Nilsson - Nyström 2009

Odorico 1990

Paschoud 2000²

Paschoud 2012
A. Mai, Scriptorum veterum nova collectio e Vaticanis codicibus, II, Romae 1827.

S. Mariev (ed.), Ioannis Antiocheni fragmenta quae supersunt omnia, Berlin 2008.

S. Mariev, Byzantine World Chronicles: Identities of Genre, in G. Greatrex - H. Elton (eds.), Shifting Genres in Late Antiquity, Ashgate 2015, 305-317.

A.M. Martolini, I frammenti dell'«Anonymus post Dionem». Pietro Patrizio nell'ambito della storiografia tardoantica e bizantina, in U. Roberto - L. Mecella (a cura di), Dalla storiografia ellenistica alla storiografia tardoantica. Aspetti, problemi, prospettive, Soveria Mannelli 2010, 209-237.

L. Mecella, L'età dei «Soldatenkaiser» nei frammenti di Pietro Patrizio e del cd. «Anonymus post Dionem», in E. Amato et al. (éd.), Les bistoriens fragmentaires de langue grecque à l'époque impériale et tardive, in corso di stampa.

L. Mecella - U. Roberto, Un maestro nell'età dei Paleologi. Massimo Planude e la tradizione sulla storia di Roma a Bisanzio, in L. Mecella - L. Russo (a cura di), Scuole e maestri dall' età antica al Medioevo, Roma 2017, 138-162.

Th. Mommsen, Über die dem Cassius Dio beigelegten Theile der Planudischen und der Constantinischen Excerpte, Hermes 6 (1872), 82-91.

I. Bekker - B.G. Niebuhr (edd.), Dexippi, Eunapii, Petri Patricii, Prisci, Malchi, Menandri Historiarum quae supersunt (CSHB), Bonnae 1829.

I. Nilsson, Discovering Literariness in the Past. Literature vs. History in the «Synopsis Chronike» of Konstantinos Manasses, in P. Odorico - P.A. Agapitos - M. Hinterberger (éd.), L'écriture de la mémoire. La littérarité de l'bistoriographie, Paris 2006, 15-31.

I. Nilsson - E. Nyström, To Compose, Read, and Use a Byzantine Text: Aspects of the Chronicle of Constantine Manasses, Byzantine and Modern Greek Studies 33 (2009), 42-60.

P. Odorico, La cultura della $\Sigma v \lambda \lambda o \gamma \eta ́$. 1. Il cosiddetto enciclopedismo bizantino. 2. Le tavole del sapere di Giovanni Damasceno, ByzZ 83 (1990), 1-21.

F. Paschoud (éd.), Zosime. Histoire nouvelle, I, Paris $2000^{2}$.

F. Paschoud, On a Recent Book by Alan Cameron: «The Last Pagans of Rome», AnTard 20 (2012), 359 . 388. 
Patzig 1894

Patzig 1896

Patzig 1897

Pérez Martín 2015

Piccolomini 1874

Pirogoff 1873

Praechter 1897

Ratti 2010

Ratti 2012

Ratti 2014

Rhoby 2014

Roberto 2005

Roberto 2016

Sathas 1894

Serruys 1907

Sotiroudis 1989
E. Patzig, Leo Grammaticus und seine Sippe, ByzZ 3 (1894), 470-497.

E. Patzig, Über einige Quellen des Zonaras I, ByzZ 5 (1896), 24-53.

E. Patzig, Über einige Quellen des Zonaras II, ByzZ 6 (1897), 322-356.

I. Pérez Martín, The Role of Maximos Planudes and Nikephoros Gregoras in the Transmission of Cassius Dio's «Roman History» and of John Xiphilinos' «Epitome», MEG 15 (2015), 175-193.

E. Piccolomini, Intorno ai «Collectanea» di Massimo Planude, RFIC 2 (1874), 101-117.

W. Pirogoff, De Eutropii Breviarii ab U.c. indole ac fontibus, Berolini 1873.

K. Praechter, Quellenkritische Studien zu Kedrenos (cod. Paris. gr. 1712), Sitzungsberichte der Bayerischen Akademie der Wissenschaften 2 (1897), 3-107.

St. Ratti, «Antiquus error». Les ultimes feux de la résistance païenne. «Scripta varia» augmentés de cinq études inédites, Turnhout 2010.

St. Ratti, Polémiques entre païens et chrétiens, Paris 2012.

St. Ratti, Fiction, déconstruction et religion. L'effacement du sens dans l'«Histoire Auguste», in C. Bertrand-Dagenbach - F. Chausson (edd.), Historiae Augustae Colloquium Nanceiense, Bari 2014, 419-433.

A. Rhoby, Quellenforschung am Beispiel der Chronik des Konstantinos Manasses, in J. Signes Codoñer - I. Pérez Martín (eds.), Textual Transmission in Byzantium: Between Textual Criticism and Quellenforschung, Turnhout 2014, 391-415.

U. Roberto (ed.), Ioannis Antiocheni fragmenta ex Historia chronica, Berlin 2005.

U. Roberto, L'interesse per Cassio Dione in Pietro Patrizio e nella burocrazia palatina dell'età di Giustiniano, in V. Fromentin et al. (éd.), Cassius Dion. Nouvelles lectures, I, Bordeaux 2016, 51-67.

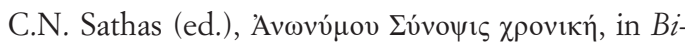
bliotheca Graeca Medii Aevii 7, Parisiis 1894, 3-556.

D. Serruys, Recherches sur l'Épitomé (Théodose de Mélitène, Léon le Grammairien, Syméon Logothète etc.), ByzZ 16 (1907), 1-51.

P. Sotiroudis, Untersuchungen zum Geschichtswerk des Johannes von Antiocheia, Hamburg 1989 (diss.). 
Tartaglia 2016

Tocci 2015

Vitiello 2014

Westerink 1966-1967-1968

Zecchini 1993

Zecchini c.d.s.
L. Tartaglia (ed.), Georgii Cedreni Historiarum Compendium, I-II, Roma 2016.

R. Tocci (ed.), Theodori Scutariotae Chronica, Berlin 2015 .

M. Vitiello, L'imperatore che amava la storia e i suoi amici. Nicomaco Flaviano e Teodosio fra «Annales» e «Historia Augusta», in C. Bertrand-Dagenbach F. Chausson (edd.), Historiae Augustae Colloquium Nanceiense, Bari 2014, 483-503.

L.G. Westerink, Le Basilikos de Maxime Planude, ByzSlav 27 (1966), 98-103; 28 (1967), 54-67; 29 (1968), 34-50 (= L.G. Westerink, Texts and Studies in Neoplatonism and Byzantine Literature: Collected Papers, Amsterdam 1980, 113-149).

G. Zecchini, Da Nicomaco Flaviano a Memmio Simmaco. La fine della storiografia classica in Occidente, in G. Zecchini, Ricerche di storiografia latina tardoantica, Roma 1993, 51-63.

G. Zecchini, Il nuovo Dexippo e l'«Historia Augusta», in B. Bleckmann - H. Brandt (edd.), Historiae Augustae Colloquium (Düsseldorf, 2015), in corso di stampa. 